\title{
Loblolly pine (Pinus taeda L.) productivity 23 years after wet site harvesting and site preparation in the lower Atlantic coastal plain
}

\author{
Charles M. Neaves III ${ }^{a}$, W. Michael Aust ${ }^{\mathrm{a}, *}$, M. Chad Bolding ${ }^{\mathrm{a}}$, Scott M. Barrett ${ }^{\mathrm{a}}$, Carl C. Trettin ${ }^{\mathrm{b}}$, \\ Eric Vance ${ }^{c}$ \\ a Department of Forest Resources and Environmental Conservation, Virginia Tech, Blacksburg, VA 24061, United States \\ ${ }^{\mathrm{b}}$ Santee-Cooper Experimental Forest, USDA Forest Service, $3734 \mathrm{Hwy}$, Cordesville, SC 29434, United States \\ ${ }^{c}$ National Council for Air and Stream Improvement, Inc. (NCASI), P.O. Box 13318, Research Triangle Park, NC 27709-3318, United States
}

\section{A R T I C L E I N F O}

\section{Article history:}

Received 10 May 2017

Received in revised form 30 June 2017

Accepted 5 July 2017

\begin{abstract}
A B S T R A C T
Ground based timber harvesting on wet sites has been linked to alteration of soil properties that may result in reduced long term site productivity. Following Hurricane Hugo in the fall of 1989, numerous salvage logging operations were conducted under high soil moisture conditions to reduce wildfire risk and salvage timber within the Francis Marion National Forest in the lower coastal plain of South Carolina. Study sites were established on wet pine flats to examine the long term effects of primary skid trails and site preparation on planted loblolly pine (Pinus taeda L.) growth. Treatment effects were analyzed as a split-plot within a randomized complete block design with 12 blocks, four levels of site preparation (none, disking, bedding, disking with bedding), and two levels of machine traffic (primary skid trail, no obvious traffic). After 23 years, bedding and disking with bedding enhanced stand density $(p<0.0001)$ and above ground stand biomass $(\mathrm{p}<0.0001)$ relative to the disking and non-site prepared treatments. None of the site preparation treatments were effective at increasing biomass of individual trees. Mean height ( $p<0.0001), D B H(p<0.0001)$, and biomass of individual trees $(\mathrm{p}<0.0001)$ were lower on primary skid trails than in non-trafficked areas. Traffic did not have a significant effect on stand density $(p<0.4662)$ or stand biomass $(p=0.1564)$. Selected soil physical properties and productivity measurements were similar for the non-site prepared treatment on and off primary skid trails, suggesting that 23 years is sufficient time for soils in wet flats to naturally recover from wet weather harvest disturbance. This study indicates that bedding may be the most efficient management practice to enhance long term stand productivity for loblolly pine on aeration-limited sites by increasing seedling survival. Minimizing the spatial extent of skid trails may increase growth of individual trees.
\end{abstract}

(c) 2017 Elsevier B.V. All rights reserved.

\section{Introduction}

\subsection{Background}

Intensively managed forest plantations are essential to satisfy the global demand for forest products with current trends in population and land use (Fox, 2000). In the southeastern United States, a substantial portion of plantation forest land is located on coastal plain wet flats, some of which are jurisdictional wetlands (Allen and Campbell, 1988; Harms et al., 1998; Stanturf et al., 2003). These forested wetlands provide a unique challenge to forest management because frequent high soil moisture conditions can accen-

\footnotetext{
* Corresponding author.

E-mail addresses: cmneaves@vt.edu (C.M. Neaves III), waust@vt.edu (W.M. Aust), bolding@vt.edu (M.C. Bolding), sbarrett@vt.edu (S.M. Barrett), ctrettin@fs.fed.us (C.C. Trettin), evance@ncasi.org (E. Vance).
}

tuate the damaging effects of equipment traffic on soil properties and site productivity (Akram and Kemper, 1979; Greacen and Sands, 1980; Hatchell et al., 1970 Miwa et al., 2004; Moehring and Rawls, 1970). It is also desirable to maintain the host of ecosystem services provided by wetlands that could be impacted by forestry practices (Richardson, 1994). Although numerous forestry professionals have recommended avoiding management practices involving heavy equipment during wet site conditions (Hatchell and Ralston, 1971; Miller et al., 2004; Moehring and Rawls, 1970; Reisinger et al., 1988), this is often not logistically or economically feasible (Miwa et al., 2004). Concerns regarding jeopardizing forest productivity via altering soil properties with ground based harvesting systems have been investigated extensively around the world (Horn et al., 2004; Makineci et al., 2007; Murphy et al., 2004; Naghdi et al., 2016; Pinard et al., 2000; Powers et al., 2005; Rab, 2004). Specifically, harvesting equipment traffic has been associated with increased bulk density, decreased 
macroporosity, and decreased hydraulic conductivity (Aust et al., 1995; Gent et al., 1983; Rab, 2004; Williamson and Neilsen, 2000), increased soil strength (Hatchell et al., 1970; Lockaby and Vidrine, 1984); water table rise (Aust et al., 1993, 1995), and reduced organic matter content (Rab, 2004). These changes in soil properties have been linked to reduced tree survival and growth (Aust et al., 1998b; Moehring and Rawls, 1970; Naghdi et al., 2016; Murphy and Firth, 2004; Wert and Thomas, 1981).

Some sites have natural mechanisms that allow sufficient long term recovery from the effects of soil disturbance such as sediment deposition, mixed clay minerology (McKee et al., 2012), high fertility, and weather patterns (Passauer et al., 2013). However, widely documented negative effects on soil properties and tree growth shortly after disturbance coupled with uncertainty of recovery potential justify implementation of site preparation as an ameliorative practice (Aust and Lea 1992; Eisenbies et al., 2004; Gent et al., 1983; Lof et al., 2012; Miller et al., 2004; Reisinger et al., 1988). Numerous site preparation techniques are available, and each method may provide a wide range of benefits that restore or enhance soil properties and productivity. Bedding is a commonly employed practice on poorly drained sites intended to increase the depth of aerated soil available to seedlings. Advantages in terms of soil physical properties and early tree growth as a result of bedding have been reported by Aust et al. (1998a), Eisenbies et al. (2004), and Hatchell (1981). Some research indicates that growth gains resulting from bedding diminish with time (Kyle et al., 2005; Wilhite and Jones, 1981; Zhao et al., 2009); however, long term effects of bedding on soil physical properties have not been widely investigated. Disking has been suggested as a method to alleviate equipment traffic induced compaction (Reisinger et al., 1988). Gent et al. (1984) concluded that disking was effective at restoring soil physical properties in the piedmont, but other studies in the coastal plain have shown disking significantly reduced macroporosity and saturated hydraulic conductivity (Aust et al., 1998b) and failed to improve slash pine (Pinus elliotti Engelm.) growth (Mann and Derr, 1970; McKee and Shoulders, 1974). Further research is needed to fully understand and predict the long term effects of wet weather harvesting disturbance and site preparation on soil properties and forest productivity on a site specific basis.

\subsection{Objectives}

The objectives of this study are to assess the effects of site preparation and primary skid trails on loblolly pine (Pinus taeda L.) growth and selected soil physical properties at age 23 years. The study also seeks to determine if loblolly pine productivity and selected soil physical property values in primary skid trails have naturally recovered to the state of a comparable, undisturbed soil at stand age 23 years.

\section{Methods}

\subsection{Study sites}

Six experimental sites were previously established in Berkeley County, South Carolina within the Francis Marion National Forest (Fig. 1). Berkeley County is in the lower Atlantic Coastal Plain physiographic province, and averages $129 \mathrm{~cm}$ of precipitation with hot summers and mild winters (NOAA, 2016).

The sites are classified as wet pine flats, characterized by minimal relief, dense argillic horizons, and a longleaf pine (Pinus palustris Mill.) and loblolly pine (Pinus taeda L.) dominated overstory. The sites were established in 1989 following the salvage logging of timber damaged by Hurricane Hugo to study the effects of wet weather primary skid trails and site preparation on soil physical properties and forest productivity (Scheerer, 1994; Tippett, 1992). Following the Hurricane, only 5-12 trees ha ${ }^{-1}$ remained standing, thus the overstory removal was similar to a clearcut harvest. Common understory species include sweet pepperbush ( $\mathrm{Cle}$ thra alnifolia), inkberry (Ilex galbra), and sweetgum (Liquidambar styraciflua).

Dominant soil series within the study sites include somewhat poorly drained Lynchburg (fine-loamy, siliceous, semiactive, thermic Aeric Paleaquults), moderately well drained Goldsboro (fineloamy, siliceous, subactive, thermic Aquic Paleudults), poorly drained Rains (fine-loamy, siliceous, semiactive, thermic Typic Paleaquults), somewhat poorly drained Wahee (fine, mixed, semiactive, thermic Aeric Endoaquults), and poorly drained Bethera (fine, mixed, semiactive, thermic Typic Paleaquults) (USDA NRCS 2016a; USDA NRCS 2016b). Each of these soils has a dense argillic horizon and a water table near the soil surface during some part of the year (USDA NRCS, 2016a).

\subsection{Experimental design}

The experiment was conducted as a split-plot within an unbalanced randomized complete block design consisting of twelve blocks with four levels of site preparation as the main plot factor (bedding, disking with bedding, disking, and no site preparation) and two machine traffic levels as the subplot factor (primary skid trail, no obvious traffic). We were unable to relocate two of the original 96 subplot experimental units; therefore, a total of 94 subplot experimental units were included in this study.

\subsection{Treatments}

The six experimental sites (two blocks per site) were salvage logged with rubber tired skidders under high soil moisture conditions during the fall and winter of 1989. Subplots within each site are distributed across approximately eight to 12 hectares. Each subplot experimental unit is $24.4 \times 6.1 \mathrm{~m}$ in size. The site preparation treatments are bedding (Bed), flat disking (Disk), flat disking with bedding (D/B), and no site preparation (None). Woody debris was mechanically removed from plots prior to treatment installation in September of 1991. Each site preparation treatment was installed on a primary skid trail (On) and in an area with no obvious traffic disturbance (Off) within each block (Tippett, 1992) (Fig. 2). Detailed maps of subplot experimental units are provided in Tippett (1992). Loblolly pine seedlings were planted within treatment plots on a $2.0 \mathrm{~m} \times 0.6 \mathrm{~m}$ spacing (8333 seedlings ha ${ }^{-1}$ ) (three rows in each subplot) in February of 1992 (Scheerer, 1994) and thinned to a $2.0 \times 1.8 \mathrm{~m}$ (2778 seedlings ha ${ }^{-1}$ ) spacing in 1996. This planting density was chosen so that we could fit multiple beds into the relatively narrow skid trails and because the experiment was originally anticipated to end after two growing seasons. While this density does not reflect a standard planting density it will allow the relative treatment effects to be evaluated. The treatment combinations of the non-located original subplot experimental units are On-Disk and Off-Disk, and are in separate blocks.

\subsection{Data collection}

All field data collection and measurements were conducted during the summer of 2015 with the exception of approximately 130 soil cores which were compromised. These cores were collected during June of 2016. The total height and diameter at breast height (DBH) of all living loblolly pine trees within plots were measured. Total above ground dry biomass was calculated using an allometric equation developed by Gonzalez-Benecke et al. (2014). Green 


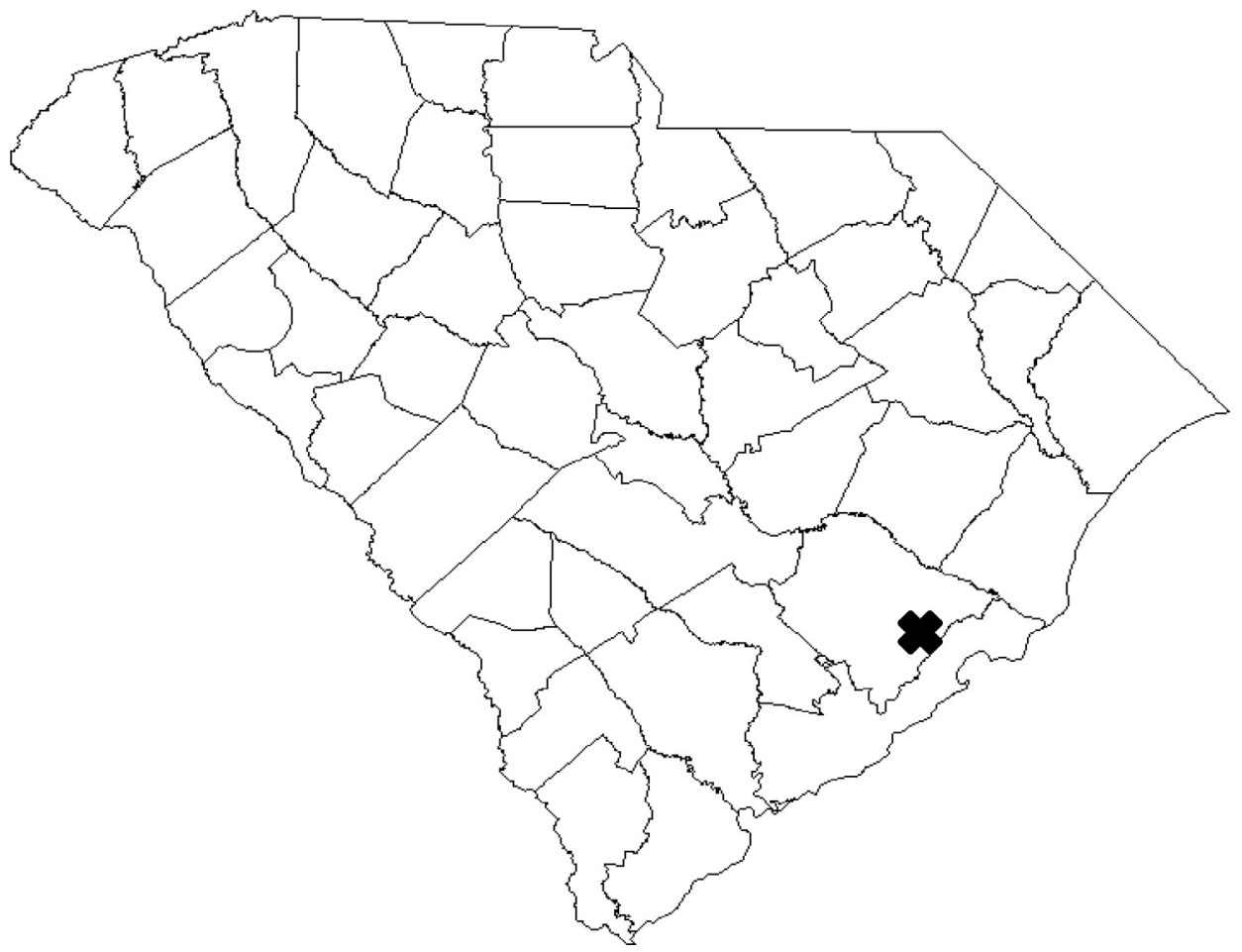

Fig. 1. Approximate location of study area in Berkeley County, South Carolina, United States.

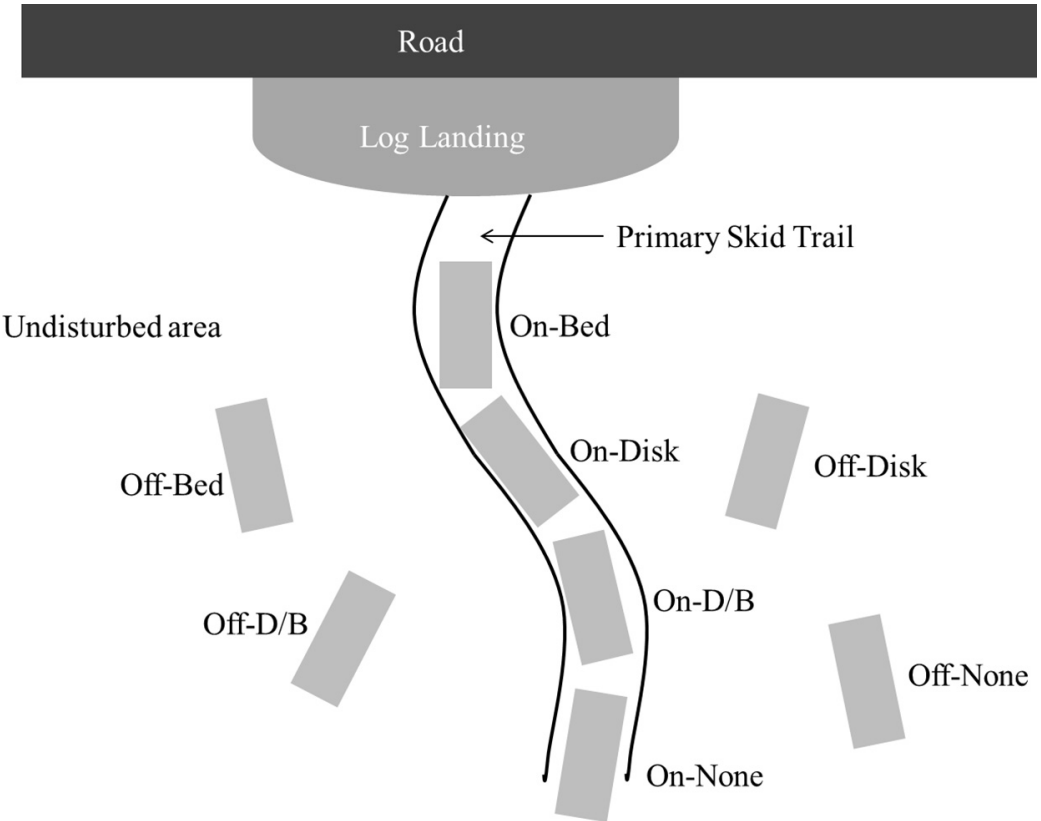

Fig. 2. Generalized layout of treatment subplots within a block.

weight of biomass was approximated by doubling the result of this equation, assuming a moisture content of $50 \%$. Stand density and biomass per hectare were calculated by scaling the number of trees and total biomass per plot, respectively, to one hectare. Eight soil cores were systematically collected in each subplot experimental unit using a double cylinder hammer driven core sampler and sealed for later analysis of saturated hydraulic conductivity (Ksat) (Klute and Dirksen, 1986), macroporosity, (Danielson and Sutherland, 1986), and bulk density (Blake and Hartge, 1986).

\subsection{Statistical analysis}

The main effects of site preparation and traffic were analyzed using standard two-way ANOVA procedures. The main effect of site preparation was applied only to biomass per hectare, stand density, bulk density, saturated hydraulic conductivity, and macroporosity due to treatment interactions. The main effect of traffic was applied to all responses. A one-way ANOVA with eight treatments (i.e. Off-Bed, Off-D/B, On-Bed) was used to analyze the 
effects of site preparation on height, $\mathrm{DBH}$, and biomass of individual trees. This analysis was also used to compare the None site preparation treatments for both traffic levels. Appropriate transformations were performed for responses exhibiting non-parametric behavior. Fisher's LSD with $\alpha=0.1$ was used for all multiple means separations. (Ott and Longnecker, 2010; Stefano, 2001). All analysis procedures were conducted using JMP Pro 13 statistical software (SAS Institute Inc, 2016).

\section{Results}

The interaction of block and site preparation was significant for stand density $(\mathrm{p}=0.0002)$, individual tree height $(\mathrm{p}=0.0001), \mathrm{DBH}$ $(\mathrm{p}<0.0001)$ and individual tree green biomass $(\mathrm{p}=0.0004)$; however, interaction plots indicate that these interactions were co-directional. All other block-treatment interactions were not significant. The interaction between site preparation and traffic was significant for individual tree height $(\mathrm{p}=0.0019), \mathrm{DBH}$ $(p=0.0346)$, and green tree biomass $(p=0.0223)$. Interaction plots for these variables indicate that traffic was masking the main effect of site preparation, so the traffic and site preparation treatment combinations were analyzed as eight different treatments.

Within the Off traffic level, Bed, D/B, and Disk had significantly greater heights than None, but were not significantly different from one another (Table 1). Disk had the greatest absolute values for DBH and individual tree biomass, and these values were significantly greater than D/B and None, but not significantly different from Bed. For the On traffic level, Bed had the greatest height and was significantly greater than Disk and None, but not D/B. D/B had the greatest average $\mathrm{DBH}$, but no treatments were significantly different. Individual tree biomass was lowest for Disk, but was not significantly lower than None. Bed and D/B individual tree biomass values were significantly greater than Disk, but not None. For the Off-None and On-None treatment comparisons, height, DBH, tree biomass, stand biomass, stand density, bulk density, Ksat and Macroporosity were not significantly different (Tables 1 and 2).

Despite significant treatment interaction, the main effect of traffic was significant on individual tree height ( $p<0.0001)$, DBH $(p<0.0001)$, and green tree biomass $(p<0.0001)$. Mean values for these variables were lower for the On treatment. Traffic did not have a significant effect on green stand biomass $(p=0.1564)$ or stand density $(\mathrm{p}=0.4662)$ (Table 3$)$.
Table 2

LS Mean values for selected soil physical properties analyzed as eight separate treatments. Values not followed by the same letter within a column are statistically different by Fisher's LSD at $\alpha=0.1$.

\begin{tabular}{lccc}
\hline $\begin{array}{l}\text { Traffic } \\
\text { Site Preparation }\end{array}$ & $\begin{array}{c}\text { Bulk Density } \\
(\text { S.E. }) \\
\left(\mathrm{Mg} \mathrm{m}^{-3}\right)\end{array}$ & $\begin{array}{c}\text { Ksat } \\
(\text { S.E. }) \\
\left(\mathrm{cm} \mathrm{h}^{-1}\right)\end{array}$ & $\begin{array}{c}\text { Macroporosity } \\
(\text { S.E. }) \\
(\%)\end{array}$ \\
\hline Off & & & \\
Bed & $1.36 \mathrm{c}$ & $43.59 \mathrm{a}$ & $12.23 \mathrm{a}$ \\
& $(0.02)$ & $(5.64)$ & $(0.57)$ \\
D/B & $1.35 \mathrm{c}$ & $27.86 \mathrm{~b}$ & $10.94 \mathrm{ab}$ \\
& $(0.02)$ & $(5.64)$ & $(0.57)$ \\
Disk & $1.43 \mathrm{ab}$ & $10.49 \mathrm{~cd}$ & $8.89 \mathrm{~d}$ \\
& $(0.02)$ & $(5.93)$ & $(0.59)$ \\
None & $1.43 \mathrm{ab}$ & $6.09 \mathrm{~d}$ & $8.26 \mathrm{~d}$ \\
& $(0.02)$ & $(5.64)$ & $(0.57)$ \\
On & & & $10.61 \mathrm{bc}$ \\
Bed & $1.43 \mathrm{ab}$ & $25.93 \mathrm{~b}$ & $(0.57)$ \\
& $(0.02)$ & $(5.64)$ & $10.84 \mathrm{~b}$ \\
D/B & $1.38 \mathrm{bc}$ & $19.68 \mathrm{bc}$ & $(0.57)$ \\
& $(0.02)$ & $(5.64)$ & $9.5 \mathrm{~cd}$ \\
Disk & $1.45 \mathrm{a}$ & $15.81 \mathrm{bcd}$ & $(0.57)$ \\
& $(0.02)$ & $(5.71)$ & $9.28 \mathrm{~cd}$ \\
None & $1.43 \mathrm{ab}$ & $19.36 \mathrm{bcd}$ & $(0.59)$ \\
& $(0.02)$ & $(5.92)$ & $<0.0001$ \\
P-Value & 0.0106 & 0.0002 & \\
\hline
\end{tabular}

Bed and D/B treatments had significantly greater green stand biomass $(p<0.0001)$ and stand density $(p<0.0001)$ than Disk and None treatments, but were not significantly different from each other. Disk and None treatments were also not significantly different in terms of green stand biomass and stand density (Table 5).

Bulk density was significantly greater for the On treatment than the Off treatment ( $\mathrm{p}=0.0862$ ) (Table 4 ). The effects of site preparation on bulk density were also significant $(p=0.0226)$. Disk had the highest bulk density, but was not significantly different from None. Bed had significantly lower bulk density than Disk, but was not significantly less than None. Bulk density for the D/B treatment was significantly less than None and Disk, but not significantly different from Bed (Table 5).

After 23 years traffic did not significantly affect Ksat $(\mathrm{p}=0.6144)$ or macroporosity $(\mathrm{p}=0.9285)$ (Table 4$)$. Bed and $\mathrm{D} / \mathrm{B}$ had significantly greater Ksat $(p=0.0567)$ and macroporosity

Table 1

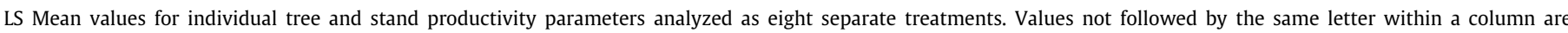
significantly different by Fisher's LSD at $\alpha=0.1$.

\begin{tabular}{|c|c|c|c|c|c|}
\hline $\begin{array}{l}\text { Traffic } \\
\text { Site Preparation }\end{array}$ & $\begin{array}{l}\text { Height } \\
\text { (S.E.) } \\
\text { (m) }\end{array}$ & $\begin{array}{l}\text { DBH } \\
(\mathrm{S} . \mathrm{E} .) \\
(\mathrm{cm})\end{array}$ & $\begin{array}{c}\text { Tree Biomass Green Weight } \\
\text { (S.E.) } \\
(\mathrm{kg})\end{array}$ & $\begin{array}{c}\text { Stand Biomass Green Weight } \\
\text { (S.E.) } \\
\left(\mathrm{Mg} \mathrm{ha}^{-1}\right)\end{array}$ & $\begin{array}{l}\text { Stand Density } \\
\quad(\text { S.E. }) \\
\left(\text { No. trees ha } \text { ha }^{-1}\right)\end{array}$ \\
\hline \multicolumn{6}{|l|}{ Off } \\
\hline Bed & $\begin{array}{l}16.0 \mathrm{a} \\
(0.25)\end{array}$ & $\begin{array}{c}19.9 \mathrm{ab} \\
(0.45)\end{array}$ & $\begin{array}{c}281.4 \mathrm{ab} \\
(14.31)\end{array}$ & $\begin{array}{c}274.1 \mathrm{ab} \\
(27.82)\end{array}$ & $\begin{array}{l}1009.1 \mathrm{a} \\
(119.54)\end{array}$ \\
\hline $\mathrm{D} / \mathrm{B}$ & $\begin{array}{l}15.8 \mathrm{ab} \\
(0.25)\end{array}$ & $\begin{array}{l}19.2 \mathrm{bc} \\
(0.45)\end{array}$ & $\begin{array}{l}266.5 \mathrm{bc} \\
(14.20)\end{array}$ & $\begin{array}{l}276.7 \mathrm{a} \\
(27.82)\end{array}$ & $\begin{array}{l}1110.0 \mathrm{a} \\
(119.54)\end{array}$ \\
\hline Disk & $\begin{array}{l}15.8 \mathrm{ab} \\
(0.40)\end{array}$ & $\begin{array}{l}20.8 \mathrm{a} \\
(0.73)\end{array}$ & $\begin{array}{l}318.9 \mathrm{a} \\
(22.87)\end{array}$ & $\begin{array}{l}148.3 \mathrm{~cd} \\
(29.23)\end{array}$ & $\begin{array}{l}458.2 \mathrm{~b} \\
(125.60)\end{array}$ \\
\hline None & $\begin{array}{l}14.7 \mathrm{~cd} \\
(0.41)\end{array}$ & $\begin{array}{c}18.7 \text { bcd } \\
(0.75)\end{array}$ & $\begin{array}{c}244.7 \mathrm{bc} \\
(23.56)\end{array}$ & $\begin{array}{l}105.7 \mathrm{~d} \\
(27.82)\end{array}$ & $\begin{array}{c}403.7 \mathrm{~b} \\
(119.54)\end{array}$ \\
\hline \multicolumn{6}{|l|}{ On } \\
\hline Bed & $\begin{array}{c}15.5 \mathrm{ab} \\
(0.24)\end{array}$ & $\begin{array}{l}18.2 \mathrm{~d} \\
(0.43)\end{array}$ & $\begin{array}{l}241.8 \mathrm{c} \\
(13.58)\end{array}$ & $\begin{array}{l}257.6 \mathrm{ab} \\
(27.82)\end{array}$ & $\begin{array}{l}1154.9 \mathrm{a} \\
(119.54)\end{array}$ \\
\hline $\mathrm{D} / \mathrm{B}$ & $\begin{array}{l}15.3 \mathrm{bc} \\
(0.26)\end{array}$ & $\begin{array}{l}18.5 \mathrm{~cd} \\
(0.48)\end{array}$ & $\begin{array}{l}244.7 \mathrm{c} \\
(15.01)\end{array}$ & $\begin{array}{l}209.9 \text { bc } \\
(27.82)\end{array}$ & $\begin{array}{c}941.8 \mathrm{a} \\
(119.54)\end{array}$ \\
\hline Disk & $\begin{array}{l}13.9 \mathrm{~d} \\
(0.36)\end{array}$ & $\begin{array}{l}17.3 \mathrm{~d} \\
(0.65)\end{array}$ & $\begin{array}{l}195.5 \mathrm{~d} \\
(20.64)\end{array}$ & $\begin{array}{l}118.0 \mathrm{~d} \\
(29.23)\end{array}$ & $\begin{array}{c}579.4 \mathrm{~b} \\
(125.60)\end{array}$ \\
\hline None & $\begin{array}{l}14.7 \mathrm{c} \\
(0.37)\end{array}$ & $\begin{array}{c}18.3 \mathrm{~cd} \\
(0.68)\end{array}$ & $\begin{array}{l}234.4 \mathrm{~cd} \\
(21.30)\end{array}$ & $\begin{array}{l}119.8 \mathrm{~d} \\
(27.82)\end{array}$ & $\begin{array}{c}493.4 \mathrm{~b} \\
(119.54)\end{array}$ \\
\hline P-Value & $<0.0001$ & 0.0012 & 0.0011 & $<0.0001$ & $<0.0001$ \\
\hline
\end{tabular}


Table 3

LS Mean values for tree and stand productivity parameters by traffic level at $\alpha=0.1$.

\begin{tabular}{|c|c|c|c|c|c|}
\hline Traffic & $\begin{array}{l}\text { Tree Height } \\
\text { (S.E.) } \\
(\mathrm{m})\end{array}$ & $\begin{array}{l}\text { Tree DBH } \\
\text { (S.E.) } \\
(\mathrm{cm})\end{array}$ & $\begin{array}{c}\text { Tree Biomass Green Weight } \\
(\text { S.E. }) \\
(\mathrm{kg})\end{array}$ & $\begin{array}{c}\text { Stand Biomass Green Weight } \\
\text { (S.E.) } \\
\left(\mathrm{Mg} \mathrm{ha}^{-1}\right)\end{array}$ & $\begin{array}{l}\text { Stand Density } \\
\quad \text { (S.E.) } \\
\text { No. trees ha }{ }^{-1}\end{array}$ \\
\hline Off & $\begin{array}{l}16.0 \mathrm{a} \\
(0.17)\end{array}$ & $\begin{array}{l}19.0 \mathrm{a} \\
(0.28)\end{array}$ & $\begin{array}{c}266.2 \mathrm{a} \\
(9.18)\end{array}$ & $\begin{array}{l}199.2 \mathrm{a} \\
(13.46)\end{array}$ & $\begin{array}{l}750.0 \mathrm{a} \\
(43.57)\end{array}$ \\
\hline On & $\begin{array}{l}15.4 \mathrm{~b} \\
(0.16)\end{array}$ & $\begin{array}{l}17.6 \mathrm{~b} \\
(0.26)\end{array}$ & $\begin{array}{c}225.1 \mathrm{~b} \\
(8.38)\end{array}$ & $\begin{array}{l}179.1 \mathrm{a} \\
(13.46)\end{array}$ & $\begin{array}{l}791.5 \mathrm{a} \\
(43.57)\end{array}$ \\
\hline
\end{tabular}

Table 4

LS Mean values of selected soil physical properties by traffic level at $\alpha=0.1$.

\begin{tabular}{cccc}
\hline Traffic & $\begin{array}{c}\text { Bulk Density } \\
(\text { S.E. })\end{array}$ & $\begin{array}{c}\text { Ksat } \\
(\text { S.E. }) \\
\left(\mathrm{Mg} \mathrm{m}^{-3}\right)\end{array}$ & $\begin{array}{c}\text { Macroporosity } \\
(\text { S.E. }) \\
(\%)\end{array}$ \\
\hline \multirow{2}{*}{ Off } & $1.39 \mathrm{a}$ & $21.98 \mathrm{a}$ & $10.11 \mathrm{a}$ \\
& $(0.01)$ & $(2.87)$ & $(0.29)$ \\
On & $1.42 \mathrm{~b}$ & $20.49 \mathrm{a}$ & $10.08 \mathrm{a}$ \\
& $(0.01)$ & $(2.91)$ & $(0.29)$ \\
\hline
\end{tabular}

( $p=0.0071$ ) than Disk and None, but are not significantly different from one another (Table 5). Disk and None also did not have significantly different Ksat or macroporosity. No significant interactions between traffic and site preparation were detected for bulk density, Ksat, and macroporosity.

\section{Discussion}

\subsection{Site preparation}

The significantly greater stand density and stand biomass for Bed and D/B treatments in the current study reflects seedling survival trends on these sites at age four years. (Aust et al., 1998c) (Table 6). The most profound benefit of bedding that enhanced seedling survival may be the increase in aerated soil depth available to roots. Inadequate aeration is a common stressor to regeneration in wet pine flats (Allen and Campbell, 1988) due to destruction of macropore space via soil disturbance (Aust et al., 1993, 1995, 1998a, 1998b; Gent et al., 1983; Greacen and Sands, 1980; Moehring and Rawls, 1970) and water table rise resulting from decreased transpiration rates (Sun et al., 2000; Xu et al., 2002) and interruption of soil drainage via machine traffic (Aust et al., 1993, 1995). Within the On treatment at age four years, Aust et al. (1998b) reported macroporosity benefits as a result of Bed and D/B treatments. Macroporosity values for None and Disk remained well under the aeration porosity threshold of $10 \%$ for adequate root growth as suggested by Vomocil and Flocker (1961). Planting beds also reduce aeration deficits by providing seedlings with greater elevation above the water table (Xu et al., 2002). Sanchez et al. (2006) further exemplified aeration benefits of bedding by noting that bedding provided greater mean stand volume of loblolly pine at age 10 years on inherently wetter parts of a study area, but not areas without excess moisture. Additional potential benefits of bedding for seedling survival include incorporation of organic matter, exposure of mineral soil, compaction alleviation, and competing vegetation control (Harms et al., 1998; Hatchell, 1981; Lof et al., 2012; Miwa et al., 2004; Reisinger et al., 1988). The initially higher seedling survival rates within the Bed and D/B treatments are probably responsible for these treatments containing approximately double the stand density and stand biomass during the 24th growing season.

Few other studies have reported long term increases in stand density and biomass as a result of bedding as dramatic as in this study. On a more fertile, intensively managed wet flat, Passauer et al. (2013) reported significantly greater loblolly pine stand density on wet harvested, bedded plots relative to wet harvested, flat planted plots at age 16 years, yet bedding only increased density by five percent. A significant difference in stand biomass was not detected. The moderate effects of bedding found by Passauer et al. (2013) may have been due to experimental controls to ensure survival through the first growing season, exceptionally dry growing seasons at ages 3-5, high soil fertility, or a combination of these factors. Andrews (1993) reported that loblolly pine stand density values for bedded and control plots were not significantly different at age 21 in a Virginia wet flat. Although stand volume per hectare was significantly greater at age 21 years on bedded plots in that study, there was no significant difference at age 33 years (Kyle et al., 2005). The persistent, dramatic response of stand density and stand biomass on our study sites contrasts long term responses shown by Andrews (1993), Kyle et al. (2005) and Passauer et al. (2013), indicating that tree responses to bedding vary with site conditions as suggested by Fox (2000) and Miwa et al. (2004).

Analyzing the effects of site preparation on individual tree measurements by traffic level revealed that the site preparation-traffic interaction resulted from varying responses to the Disk treatment. Disk has a positive effect on individual tree metrics relative to

Table 5

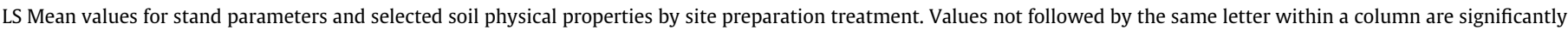
different by Fisher's LSD at $\alpha=0.1$.

\begin{tabular}{|c|c|c|c|c|c|}
\hline Site Preparation & $\begin{array}{c}\text { Stand Biomass Green Weight } \\
\text { (S.E.) } \\
\left(\mathrm{Mg} \mathrm{ha}^{-1}\right)\end{array}$ & $\begin{array}{l}\text { Stand Density } \\
\text { (S.E.) } \\
\text { (No. Trees ha }{ }^{-1} \text { ) }\end{array}$ & $\begin{array}{l}\text { Bulk Density } \\
\text { (S.E.) } \\
\left(\mathrm{Mg} \mathrm{m}^{-3}\right)\end{array}$ & $\begin{array}{c}\text { Ksat } \\
(\text { S.E. }) \\
\left(\mathrm{cm} \mathrm{h}^{-1}\right)\end{array}$ & $\begin{array}{l}\text { Macroporosity } \\
\text { (S.E.) } \\
(\%) \\
\end{array}$ \\
\hline Bed & $\begin{array}{l}265.8 \mathrm{a} \\
(20.91)\end{array}$ & $\begin{array}{l}1082.0 \mathrm{a} \\
(107.37)\end{array}$ & $\begin{array}{c}1.39 \mathrm{bc} \\
(0.02)\end{array}$ & $\begin{array}{c}34.76 \mathrm{a} \\
(4.41)\end{array}$ & $\begin{array}{c}11.42 \mathrm{a} \\
(0.44)\end{array}$ \\
\hline $\mathrm{D} / \mathrm{B}$ & $\begin{array}{l}243.2 \mathrm{a} \\
(20.91)\end{array}$ & $\begin{array}{l}1025.9 \mathrm{a} \\
(107.37)\end{array}$ & $\begin{array}{l}1.36 \mathrm{c} \\
(0.02)\end{array}$ & $\begin{array}{c}23.77 \mathrm{a} \\
(4.41)\end{array}$ & $\begin{array}{c}10.89 \mathrm{a} \\
(0.44)\end{array}$ \\
\hline Disk & $\begin{array}{l}129.8 \mathrm{~b} \\
(22.59)\end{array}$ & $\begin{array}{c}516.8 \text { b } \\
(115.79)\end{array}$ & $\begin{array}{l}1.43 \mathrm{a} \\
(0.02)\end{array}$ & $\begin{array}{c}13.80 \mathrm{~b} \\
(4.71)\end{array}$ & $\begin{array}{l}9.27 \mathrm{~b} \\
(0.46)\end{array}$ \\
\hline None & $\begin{array}{l}112.8 \text { b } \\
(20.91)\end{array}$ & $\begin{array}{c}448.5 \mathrm{~b} \\
(107.37)\end{array}$ & $\begin{array}{c}1.42 \mathrm{ab} \\
(0.02)\end{array}$ & $\begin{array}{c}11.92 \mathrm{~b} \\
(4.60)\end{array}$ & $\begin{array}{l}8.27 \mathrm{~b} \\
(0.45)\end{array}$ \\
\hline
\end{tabular}


Table 6

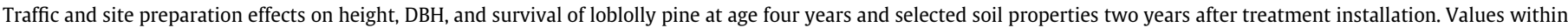

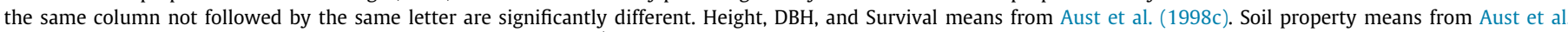
(1998b). Means separation performed using Fisher's LSD at $\alpha=0.1^{*}$ or $\alpha=0.05^{+}$.

\begin{tabular}{|c|c|c|c|c|c|c|}
\hline $\begin{array}{l}\text { Traffic } \\
\text { Site Preparation }\end{array}$ & Height $^{+}(\mathrm{m})$ & $\mathrm{DBH}^{+}(\mathrm{cm})$ & Survival $^{+}(\%)$ & Bulk Density $^{*}\left(\mathrm{Mg} \mathrm{m}^{-3}\right)$ & Macroporosity $^{*}(\%)$ & Ksat $^{*}\left(\mathrm{~cm} \mathrm{~h}^{-1}\right)$ \\
\hline \multicolumn{7}{|l|}{ Off } \\
\hline Bed & $2.7 \mathrm{a}$ & $2.4 \mathrm{a}$ & $85 a$ & $1.25 \mathrm{a}$ & $14.3 \mathrm{c}$ & $2.9 \mathrm{bc}$ \\
\hline $\mathrm{D} / \mathrm{B}$ & $2.5 \mathrm{a}$ & $3.1 \mathrm{a}$ & $84 \mathrm{a}$ & $1.25 \mathrm{a}$ & $14.6 \mathrm{c}$ & $2.2 \mathrm{~b}$ \\
\hline Disk & $1.5 \mathrm{~b}$ & $1.2 \mathrm{~b}$ & $68 \mathrm{~b}$ & $1.23 \mathrm{a}$ & $12.5 \mathrm{~b}$ & $0.5 \mathrm{a}$ \\
\hline None & $1.6 \mathrm{~b}$ & $1.6 \mathrm{~b}$ & $64 \mathrm{~b}$ & $1.22 \mathrm{a}$ & $15.1 \mathrm{c}$ & $5.0 \mathrm{c}$ \\
\hline \multicolumn{7}{|l|}{ On } \\
\hline Bed & $2.5 \mathrm{a}$ & $3.0 \mathrm{a}$ & $87 \mathrm{a}$ & $1.29 \mathrm{~b}$ & $13.3 \mathrm{bc}$ & $1.4 \mathrm{ab}$ \\
\hline $\mathrm{D} / \mathrm{B}$ & $2.3 \mathrm{a}$ & $2.7 \mathrm{a}$ & 82 a & $1.34 \mathrm{~b}$ & $9.9 \mathrm{~b}$ & $0.5 \mathrm{a}$ \\
\hline Disk & $1.5 \mathrm{~b}$ & $1.1 \mathrm{~b}$ & $71 \mathrm{~b}$ & $1.38 \mathrm{~b}$ & $2.7 \mathrm{a}$ & $0.5 \mathrm{a}$ \\
\hline None & $1.4 \mathrm{~b}$ & $0.9 \mathrm{~b}$ & $66 \mathrm{~b}$ & $1.33 \mathrm{~b}$ & $2.6 \mathrm{a}$ & $1.0 \mathrm{a}$ \\
\hline
\end{tabular}

None for the Off traffic level. For the On traffic level, Disk has negative effects on individual tree metrics, although the effect is only significant for height (Table 1). It is possible that the Off-Disk treatment incorporated organic matter into the soil profile (Miwa et al., 2004), providing a slowly available source of nutrients that benefitted individual tree growth relative to Off-None. Organic matter can be displaced on primary skid trails (Naghdi et al., 2016; Rab, 2004), such that none is left behind and incorporated during site preparation.

Generally, the effects of Bed and D/B on individual tree metrics are very similar at age 23 years for both traffic levels. For the Off traffic level, the significant height advantage of Bed and D/B over None is likely the result of more favorable bulk density, Ksat, and macroporosity (Tables 1 and 2). For the On traffic level, only Bed provides significant height gains over None. It is surprising that $\mathrm{D} / \mathrm{B}$ does not also result in significant gains, given its soil property similarities to Bed. At both traffic levels, neither Bed nor D/B provided a significant advantage in terms of $\mathrm{DBH}$ or individual tree biomass, probably due to the greater stand density of Bed and $\mathrm{D} / \mathrm{B}$. It is important to note that Bed and D/B provided significant height and DBH advantages relative to Disk and None at age 4 years, although biomass was not calculated (Aust et al., 1998c) (Table 6). At age 23 years, the lack of significantly greater individual tree biomass coupled with only a slight increase in height suggests that the growth of individual trees is following a type $C$ response for Bed and $\mathrm{D} / \mathrm{B}$, which connotes early growth gains that diminish with time (Morris and Lowery, 1988). This long term response type has also been reported in wet flats with loblolly pine in Virginia (Kyle et al. (2005) and slash pine in Florida and Georgia (Wilhite and Jones, 1981; Zhao et al., 2009). Relationships between water table elevation and transpiration rates likely contribute to this response. Water tables rise following harvest due to reduced transpiration rates (Sun et al., 2000; Xu et al., 2002) and soil disturbance (Aust et al., 1993, 1995) in forested wetlands, imposing excess moisture stress on seedlings. Bedding alleviates this limitation for seedling growth. As seedlings become established and transpiration rates are restored on non-bedded sites, the excess moisture stress will eventually be overcome (Kyle et al., 2005). Additionally, trees may rapidly deplete nutrients that become concentrated in beds (Tiarks and Haywood, 1996) leading to rapid early growth that slows with time. Non-bedded plots are expected to have a more uniform distribution of nutrients and organic matter, and therefore, a more consistent growth rate through the rotation.

It is interesting that soil bulk density values at stand age two years (Aust et al., 1998b) were lower than those at stand age 23 years. It is likely that incorporation of organic debris and litter left on site after salvage logging reduced bulk densities. Over time, a portion organic matter may have oxidized, allowing mineral soil particles to settle into a smaller volume. There may also be a similar influence of soil settling in the Bed and D/B treatments. Differences in systematic subsample collection are probably another contributing factor to the apparent increase in bulk density.

At age 23 years, the study indicates that bedding and disking with bedding greatly enhanced the amount of biomass accrued in the stand, likely due to greater initial survival, but no site preparation appeared to be advantageous for the biomass of individual trees. Disking with bedding shows no significant advantages to bedding in terms of individual tree growth, stand measurements and selected soil physical properties, yet is more expensive to implement. Thus, bedding is recommended as the most costeffective method to ensure adequate survival, increasing long term stand productivity for loblolly pine in disturbed or undisturbed soils when excess moisture is a stressor to survival early in the rotation.

\subsection{Traffic}

It is not surprising that traffic on primary skid trails reduced mean individual heights, $\mathrm{DBH}$, and biomass of loblolly pine (Table 3). Numerous researchers have documented reduced individual tree metrics in skid roads and harvest areas disturbed by heavy equipment under wet and dry trafficking conditions (e.g. Carter et al., 2006; Lockaby and Vidrine, 1984; Moehring and Rawls, 1970; Murphy et al., 2004; Naghdi et al., 2016; Wert and Thomas, 1981). In contrast to this study, several studies have shown decreased whole stand productivity as well (Hatchell et al., 1970; Lockaby and Vidrine, 1984; Murphy and Firth, 2004; Wert and Thomas, 1981). Reduced stand productivity was found by Hatchell et al. (1970) and Lockaby and Vidrine (1984) at stand age one and five years, respectively, which is probably not long enough to allow soil recovery. Decreased stand volume has been reported to persist through ages 21 and 37 years by Murphy et al. (2004) in New Zealand and Wert and Thomas (1981) in Oregon, United States, respectively, although both of these studies have inherently different climate and soil conditions than our study sites. Additionally, treatments in Murphy et al. (2004) included topsoil and litter removal which may have exacerbated the effects of compaction. Our study results were consistent with those of Sanchez et al. (2006) on similar soils in North Carolina, who found that stand volume was not significantly decreased by intentional compaction.

Reduced tree growth and stand productivity following harvest related disturbance, particularly on wet sites, is typically linked to increased bulk density, reduced macroporosity, and reduced hydraulic conductivity (Aust et al., 1998b; Gent et al., 1983; Lockaby and Vidrine, 1984; Moehring and Rawls, 1970; Reisinger et al., 1988). At our study sites, bulk density, macroporosity, and 
Ksat in On plots have generally recovered to values similar to Off plots despite being substantially different following disturbance (Aust et al., 1998b). The only soil property remaining significantly different by traffic is bulk density, although it is only $0.03 \mathrm{Mg} \mathrm{m}^{-3}$ greater for the On treatment. This small difference in bulk density is unlikely to have a substantial influence on tree growth, and the value of $1.42 \mathrm{Mg} \mathrm{m}^{-3}$ (Table 2) is below the generally accepted root limiting bulk density for the surface texture of all soil series at our study sites (Daddow and Warrington, 1983). The recovery of soil properties degraded by wet weather harvesting is consistent with the conclusions of Lang et al. (2016) after 17 years. Despite these soil property similarities, individual tree metrics in the On plots remain inferior to those in Off plots, perhaps because trees were subjected to more stressful growing conditions during the time required for soil properties to recover. This result contradicts the conclusion that wet weather harvesting is generally not harmful to loblolly pine growth at age 16 found by Passauer et al. (2013). This may be because measurements by Passauer et al. (2013) were taken across the entire harvest area, representing lower traffic intensity than primary skid trails alone. The higher stand density of On offsets the lesser individual tree biomass such that stand biomass is not significantly different by traffic level. It is unclear if the difference in stand density is the cause or effect of individual tree biomass. However, stand density is not significantly different by traffic, reflecting the trend in survival at age four years (Aust et al., 1998b, 1998c) (Table 6).

\subsection{Recovery of primary skid trails}

Four years after wet weather harvest, bulk density was significantly greater and macroporosity and Ksat were significantly lower for On-None compared to Off-None. Likewise, seedling height and diameter was lower through age two years for On-None compared to Off-None (Aust et al., 1998b). At stand age 23 years, the similar values for Off-None and On-None for all metrics suggest that skid trail soil properties and productivity levels have recovered to the state of an undisturbed soil without site preparation. Natural soil recovery mechanisms active at the sites include wet-dry cycles, bioturbation by soil fauna, and rooting activity. Shrink-swell potentials are generally low at the sites and minerology is predominantly siliceous (Long, 1980). Wet-dry cycles accelerate aggregate formation while soil fauna and rooting activity form voids in soil and incorporate organic matter. Each of these mechanisms could contribute to increased macroporosity and decreased bulk density (Larson and Allamaras, 1971; Miwa et al., 2004). It is possible that wet-dry cycles had substantial influence on recovery due to frequent observation of cyclic ponding and dry-down that occurred while field work was in progress. Abundance of active soil-burrowing organisms was confirmed during data collection. The efficacy of natural recovery mechanisms at these sites implies that site preparation is not necessary to restore soil and loblolly pine productivity of primary skid trails to a level comparable to undisturbed soils in the long term. Natural recovery of site productivity at stand age 16 years (Passauer et al., 2013) and soil properties 17 years posttreatment (Lang et al., 2016) was reported on a similar but more fertile site with greater shrink-swell potential. Rab (2004) found the deleterious effects of primary skid trails on macroporosity and bulk density to persist through ten years on an upland site. This may suggest that natural recovery mechanisms are more effective in wet flats, perhaps due to cyclic hydrologic fluxes. Additionally, high bulk density limitations may be more easily ameliorated by rooting activity in wetlands because mechanical resistance to root growth is inversely correlated with soil moisture (Busscher et al., 1997). The ability of roots to grow outside of primary skid trials within a few years of planting (Aust et al., 1998b) may have also helped productivity of loblolly pine recover.

\section{Conclusions}

At these sites, bedding and disking with bedding were effective site preparation treatments that greatly enhanced loblolly pine stand productivity, but not individual tree growth parameters at age 23 years. Stand productivity for loblolly pine was increased due to greater seedling survival in bedding and disking with bedding treatments. Bedding is a more efficient management practice than disking with bedding because both treatments provide similar soil properties and loblolly pine productivity, but disking with bedding is more expensive. Ensuring sufficient seedling survival is the foremost critical challenge forest managers encounter when establishing a stand, and practices intended to improve tree growth may be an inefficient allocation of resources if initial seedling survival is poor. It is therefore recommended that bedding be implemented in intensive plantation forestry where poor soil aeration may threaten regeneration of an adequately stocked stand. When all site preparation treatments are considered, rubber-tired skidder trafficking during wet weather did not decrease overall stand productivity, yet the wet weather skidding treatments had decreased individual tree growth parameters, despite apparent long term recovery of selected soil physical properties. Thus the common best management practice of avoiding wet-weather operations and limiting the spatial extent of soil disturbance in order to prevent soil and productivity degradation is supported by our individual trees growth data, but not the stand growth data. Such differentiation of individual tree biomass versus stand biomass could be factors of interest depending on whether management goals were total biomass production or more valuable wood products. Overall, site preparation is not necessary, if it is strictly intended to restore primary skid trail soil properties and loblolly pine productivity levels to those of undisturbed, non-site prepared soils, due to the efficacy of natural recovery mechanisms over the course of 23 years for these particular forested wetlands, but bedding treatments offered advantages for production of loblolly pine when compared to the none site preparation treatment.

\section{Acknowledgements}

This research received financial and/or logistical support from the McIntire-Stennis Program of the National Institute of Food and Agriculture, USDA; the National Council for Air and Stream Improvement Inc. (NCASI); The USDA Forest Service Southern Research Station Santee Experimental Forest personnel; the Virginia Tech Forest Operations and Business Research Cooperative; and the Virginia Tech Department of Forest Resources and Environmental Conservation.

\section{References}

Allen, H.L., Campbell, R.G., 1988. Wet site pine management in the southeastern United States. In: Hook, D.D. et al. (Eds.), The Ecology and Management of Wetlands, vol. 2. Timber Press, Portland, OR. pp. 173-184.

Akram, M., Kemper, W.D., 1979. Infiltration of soils as affected by the pressure and water content at the time of compaction. Soil Sci. Soc. Am. J. 43, 1080-1086.

Andrews, L.M., 1993. Loblolly pine response to drainage and fertilization of hydric soils. MS Thesis, Virginia Polytechnic Institute and State University, Blacksburg, VA. 158 p.

Aust, W.M., Burger, J.A., Carter, E.A., Preston, D.P., Patterson, S.C., 1998a. Visually determined disturbance classes used as indices of forest harvesting disturbance. South. J. Appl. For. 22 (4), 245-250.

Aust, W.M., Burger, J.A., McKee Jr., W.H., Scheerer, G.A., Tippett, M.D., 1998b. Bedding and fertilization ameliorate effects of designated wet-weather skid trails after four years for loblolly pine (Pinus taeda) plantations. South. J. Appl. For. 22 (4), 222-226.

Aust, W.M., Burger, J.A., Scheerer, G.A., Tippett, M.D., McKee Jr., W.H., 1998c. Amelioration of compacted and rutted skid trails on wet pine flats: fourth-year results. In: Thomas, W.A. (Ed.), Proceedings of the Ninth Biennial Southern Silvicultural Research Conference. USDA Forest Service Southern Research Station Gen. Tech. Rep. SRS-20, Asheville, NC. pp. 386-391 (p. 628). 
Aust, W.M., Lea, R., 1992. Comparative effects of aerial and ground logging on soil properties in a tupelo-cypress wetland. For. Ecol. Manage. 50, 57-73.

Aust, W.M., Reisinger, T.W., Burger, J.A., Stokes, B.J., 1993. Soil physical and hydrological changes associated with logging a wet pine flat with wide-tired skidders. South. J. Appl. For. 17 (1), 22-25.

Aust, W.M., Tippett, M.D., Burger, J.A., McKee Jr., W.H., 1995. Compaction and rutting during harvesting affect better drained soils more than poorly drained soils on wet pine flats. South. J. Appl. For. 18 (2), 72-77.

Blake, G.R., Hartge, K.H., 1986. Bulk density. In: Klute, A. (Ed.), Methods of Soil Analysis: Part 1, Physical and Mineralogical Methods, second ed. American Society of Agronomy, Soil Science Society of America Publisher, Madison, Wisconsin, pp. 363-376 (1188p).

Busscher, W.J., Bauer, P.J., Camp, C.R., Sojka, R.E., 1997. Correction of cone index for soil water content differences in a coastal plain soil. Soil Tillage Res. 43, 205217.

Carter, M.C., Dean, T.J., Wang, Z., Newbold, R.A., 2006. Impacts of harvesting and postharvest treatments on soil bulk density, soil strength, and early growth of Pinus taeda in the Gulf coastal plain: a long-term soil productivity affiliated study. Can. J. For. Res. 36, 601-614.

Dadow, R.L., Warrington, G.E., 1983. Growth-limiting bulk densities as influenced by soil texture. USDA Forest Service Watershed systems develop group report WSDG-TN-00005, Fort Collins, CO, 17p.

Danielson, R.E., Sutherland, P.L., 1986. Porosity. In: Klute, A. (Ed.), Methods of Soil Analysis: Part 1, Physical and Mineralogical Methods, second ed. American Society of Agronomy, Soil Science Society of America Publisher. Madison, Wisconsin, pp. 443-462 (1188p).

Eisenbies, M.H., Burger, J.A., Aust, W.M., Patterson, S.C., 2004. Loblolly pine response to wet-weather harvesting on wet flats after 5 years. Water Air Soil Pollut. 4, 217-233.

Fox, T.R., 2000. Sustained productivity in intensively managed forest plantations. For. Ecol. Manage. 138, 187-202.

Gent Jr., J.A., Ballard, R., Hassan, A.E., 1983. The impact of harvesting and site preparation on physical properties of lower coastal plain soils. Soil Sci. Soc. Am. J. 47, 595-598.

Gent Jr., J.A., Ballard, R., Hassan, A.E., Cassel, D.K., 1984. Impact of harvesting and site preparation on physical properties of piedmont forest soils. Soil Sci. Soc. Am. J. $48,173-177$

Gonzalez-Benecke, C.A., Gezan, S.A., Albaugh, T.J., Allen, H.L., Burkhart, H.E., Fox, T. R., Jokela, E.J., Maier, C.A., Martin, T.A., Rubilar, R.A., Samuelson, L.J., 2014. Local and general above-stump biomass functions for loblolly pine and slash pine trees. For. Ecol. Manage. 334, 254-276.

Greacen, E.L., Sands, R., 1980. Compaction of forest soils. A review. Aust. J. Soil Res. $18,163-189$.

Harms, W.R., Aust, W.M., Burger, J.A., 1998. Wet flatwoods. In: Messina, M.G., Connor, W.H. (Eds.), Southern Forested Wetlands Ecology and Management. Lewis Publishers, New York, pp. 421-444.

Hatchell, G.E., 1981. Site preparation and fertilizer increase pine growth on soils compacted in logging. South. J. Appl. For. 5, 79-83.

Hatchell, G.E., Ralston, C.W., 1971. Natural recovery of surface soils disturbed in logging. Tree Planter's Notes. 22 (2), 5-9.

Hatchell, G.E., Ralston, C.W., Foil, R.R., 1970. Soil disturbances in logging. J. For. 68, $772-775$.

Horn, R., Vossbrink, J., Becker, S., 2004. Modern forestry vehicles and their impacts on soil physical properties. Soil Tillage Res. 79, 207-219.

Klute, A., Dirksen, C., 1986. Hydraulic conductivity. In: Klute, A. (Ed.), Methods of Soil Analysis: Part 1, Physical and Mineralogical Methods, second ed. American Society of Agronomy, Soil Science Society of America Publisher, Madison, Wisconsin, pp. 687-734 (1188p).

Kyle, K.H., Andrews, L.J., Fox, T.R., Aust, W.M., Burger, J.A., Hansen, G.H., 2005. Long term effects of drainage, bedding, and fertilization on growth of loblolly pine (Pinus taeda L.) in the coastal plain of Virginia. South. J. Appl. For. 29 (4), 205214.

Lang, A.J., Cristan, R., Aust, W.M., Bolding, M.C., Strahm, B.D., Vance, E.D., Roberts Jr., E.T., 2016. Long-term effects of wet and dry site harvesting on soil physical properties mitigated by mechanical site preparation in coastal plain loblolly pine (Pinus taeda) plantations. For. Ecol. Manage. 359, 162-173.

Larson, W.E., Allamaras, R.R., 1971. Management factors and natural forces as related to compaction. In: Barnes, K.K. et al. (Eds.), Compaction of Agricultural Soils. ASAE, Joseph, MI, pp. 367-427.

Lockaby, B.G., Vidrine, C.G., 1984. Effect of logging equipment traffic on soil density and growth and survival of young loblolly pine. South. J. Appl. For. 8 (2), 109112.

Lof, M., Dey, D.C., Navarro, R.M., Jacobs, D.F., 2012. Mechanical site preparation for forest restoration. New Forest. 43, 825-848.

Long, B.M., 1980. Soil Survey of Berkeley County. USDA Natural Resources Conservation Service, South Carolina.

Makineci, E., Demir, M., Yilmaz, E., 2007. Long-term harvesting effects on a skid road in a fir (Abies bornmulleriana Mattf.) plantation forest. Build. Environ. 42, 15381543.

Mann Jr., W.F., Derr, H.J., 1970. Response of planted loblolly and slash pine to disking on a poorly drained site. USDA for. Serv. Res. Note SO-110. 3p.

McKee, S.E., Aust, W.M., Seiler, J.R., Strahm, B.D., Schilling, E.B., 2012. Long-term site productivity of a tupelo-cypress swamp 24 years after harvest disturbances. For. Ecol. Manage. 265, 172-180.

McKee Jr., W.H., Shoulders, E., 1974. Slash pine biomass response to site preparation and soil properties. Soil Sci. Soc. Am. Proc. 38, 144-148.
Miller, R.E., Colbert, S.R., Morris, L.A., 2004. Effects of heavy equipment on physical properties of soils and on long-term productivity: a review of literature and current research. Tech. Bull. No. 887, National Council for Air and Stream Improvement, Research Triangle Park, NC.

Miwa, M., Aust, W.M., Burger, J.A., Patterson, S.C., Carter, E.A., 2004. Wet-weather timber harvesting and site preparation effects on coastal plain sites: a review. South. J. Appl. For. 28 (3), 137-151.

Moehring, D.M., Rawls, I.W., 1970. Detrimental effects of wet weather logging. J. For 68, 166-167.

Morris, L.A., Lowery, R.F., 1988. Influence of site preparation on soil conditions affecting stand establishment and tree growth. South. J. Appl. For. 12 (3), 170178.

Murphy, G., Firth, J., 2004. Soil disturbance impacts on early growth and management of radiata pine trees in New Zealand. West. J. Appl. For. 19 (2), 109-116.

Murphy, G., Firth, J.G., Skinner, M.F., 2004. Long-term impacts of forest harvesting related soil disturbance on log product yields and economic potential in a New Zealand forest. Silva Fennica 38 (3), 279-289.

Naghdi, R., Labelle, E.R., Solgi, A., Zenner, E.K., 2016. Influence of ground-based skidding on physical and chemical properties of forest soil and their effects on maple seedling growth. Eur. J. For. Res. http://dx.doi.org/10.1007/s10342-0160986-3.

National Oceanic and Atmospheric Administration National Centers for Environmental Information, 2016. Climate Data Online. Accessed March 4, 2016.

Ott, R.L., Longnecker, M., 2010. An Introduction to Statistical Methods and Data Analysis. Duxbury Press, Belmont, CA, p. 1296.

Passauer, D.P., Aust, W.M., Bolding, M.C., Strahm, B.D., Burger, J.M., Patterson, S.C., Vance, E., Roberts Jr., E.T., 2013. Potential above-ground biomass losses from severe soil rutting during wet weather timber harvest of coastal plain loblolly pine (Pinus taeda) plantations mitigated by mechanical site preparation. For. Ecol. Manage. 307, 266-273.

Pinard, M.A., Barker, M.G., Tay, J., 2000. Soil disturbance and post-logging forest recovery on bulldozer paths in Sabah, Malaysia. For. Ecol. Manage. 130, 213 225.

Powers, R.F., Scott, D.A., Sanchez, F.G., Voldseth, R.A., Page-Dumroese, D., Elioff, JD. Stone, DM., 2005. The North American long-term soil productivity experiment: findings from the first decade of research. For. Ecol. Manage. 220, 31-50.

Rab, M.A., 2004. Recovery of soil physical properties from compaction and soil profile disturbance caused by logging of native forest in Victorian Central Highlands, Australia. For. Ecol. Manage. 191, 329-340.

Reisinger, T.W., Simmons, G.L., Pope, P.E., 1988. The impact of timber harvesting on soil properties and seedling growth in the South. South. J. Appl. For. 12, 58-67.

Richardson, C.J., 1994. Ecological functions and human values in wetlands: a framework for assessing forestry impacts. Wetlands 14 (1), 1-9.

Sanchez, F.G., Scott, D.A., Ludovici, K.H., 2006. Negligible effects of severe organic matter removal and soil compaction on loblolly pine growth over 10 years. For. Ecol. Manage. 227, 145-154.

SAS Institute Inc, 2016. Using JMP 13. SAS Institute Inc, Cary, NC.

Scheerer, G.A., 1994. Mitigation of harvesting disturbances on a forested wetland in the South Carolina lower coastal plain. M.S. Thesis, Dep. of For., VPI\&SU, Blacksburg, VA, 125p.

Stanturf, J.A., Kellison, R.C., Broerman, F.S., Jones, S.B., 2003. Productivity of southern pine plantations: where are we and how did we get there?. J. For. 101 (3), 2631.

Stefano, J.D., 2001. Power analysis and sustainable forest management. For. Ecol. Manage. 154, 141-153.

Sun, G., Riekerk, H., Kornhak, L.V., 2000. Ground-water table rise after forest harvesting on cypress-pine flatwoods in Florida. Wetlands 20 (1), 101-112.

Tiarks, A.E., Haywood, J.D., 1996. Site preparation and fertilization effects on growth of slash pine for two rotations. Soil Sci. Soc. Am. J. 60, 1654-1663.

Tippett, M.D., 1992. Impacts of timber harvesting on soil physical properties in wetlands. M.S. thesis, Dep. of For., VPI\&SU, Blacksburg, VA, 165p.

USDA Natural Resources Conservation Service, 2016a. Official Soil Series Descriptions. <https://soilseries.sc.egov.usda.gov/osdname.aspx>.

USDA Natural Resources Conservation Service, 2016b. USDA NRCS Web Soil Survey. <http://websoilsurvey.sc.egov.usda.gov/App/WebSoilSurvey.aspx>.

Vomocil, J.A., Flocker, W.J., 1961. Effect of soil compaction on storage and movement of soil air and water. Trans. ASAE 4, 242-246.

Wert, S., Thomas, B.R., 1981. Effects of skid roads on diameter, height, and volume growth in douglas-fir. Soil Sci. Soc. Am. J. 45, 629-632.

Wilhite, L.P., Jones Jr., E.P., 1981. Bedding effects in maturing slash pine stands. South. J. Appl. For. 5 (1), 24-27.

Williamson, J.R., Neilsen, W.A., 2000. The influence of forest site on rate and extent of soil compaction and profile disturbance of skid trails during ground-based harvesting. Can. J. For. Res. 30, 1196-1205.

Xu, Y.J., Burger, J.A., Aust, W.M., Patterson, S.C., Miwa, M., Preston, D.P., 2002 Changes in surface water table depth and soil physical properties after harvest and establishment of loblolly pine (Pinus taeda) in Atlantic Coastal plain wetlands of South Carolina. Soil Tillage Res. 63, 109-121.

Zhao, D., Kane, M., Borders, B., Harrison, M., 2009. Long-term effects of site preparation treatments, complete competition control, and repeated fertilization on growth of slash pine in the flatwoods of the Southeastern United States. For. Sci. 55 (5), 403-410. 